\title{
Metabolic and Functional Profile of Premenopausal Women With Metabolic Syndrome After Training With Elastics as Compared to Free Weights
}

Biological Research for Nursing

$1-8$

(c) The Author(s) 2016

Reprints and permission:

sagepub.com/journalsPermissions.nav DOI: $10.1177 / 1099800416674307$

brn.sagepub.com

(9SAGE

\author{
Jorge Flandez, PhD', Noelia Belando, $\mathrm{PhD}^{2}$, Pedro Gargallo, $\mathrm{MSc}^{2}$, \\ Julio Fernández-Garrido, $\mathbf{P h D}^{3}$, Ronald $\mathrm{A}$. Vargas-Foitzick, $\mathbf{P h D}^{4}$, \\ Jose Devis-Devis, $\mathrm{PhD}^{5}$, and Juan C. Colado, PhD $^{2,5}$
}

\begin{abstract}
The aim of this study was to compare the effects of a strength training program (STP) using free weights (FW) versus elastic tubing (ET) in 62 premenopausal, sedentary women diagnosed with metabolic syndrome (MS). Participants were randomly assigned to the FW or ET experimental group (EG) or a control group whose members remained sedentary. Members of each EG followed their assigned STP for 12 weeks, and biomarkers (BMs) related to MS and motor function (MF) parameters were evaluated. Both EGs showed a significant reduction in C-reactive protein level and a positive trend in the other BMs. Almost all MF parameters increased significantly in both EGs. No positive changes were found in the CG. These results indicate that the implementation of an STP, with either FW or ET, improves both metabolic health and MF and should be considered part of the basic approach to health care in women.
\end{abstract}

\section{Keywords}

strength, elastic tubing, free weights, cardiovascular risk

Women between 45 and 55 years old are close to the end of their reproductive lives. The hormonal changes associated with menopause contribute to the development of metabolic risk factors such as dyslipidemia, insulin resistance, and increased abdominal fat (Orsatti, Nahas, Maesta, Nahas-Neto, \& Burini, 2008), which are all possible triggers for various cardiovascular risk factors (Huebschmann et al., 2015). Conclusive scientific evidence demonstrates a relationship between physical inactivity and the presence of risk factors associated with metabolic syndrome (MS) and obesity (J. W. Kim \& Kim, 2012). Moreover, regular exercise may reduce the loss of strength that is common among women in this age-group (Lauretani et al., 2003), in turn enhancing their physical performance (Clark \& Manini, 2008) and metabolic health (Gudmundsdottir, Flanders, \& Augestad, 2013).

Several studies have shown the effectiveness of resistance training in reducing cardiovascular risk and dynapenia in women of different ages, including the menopausal agegroup (Colado, Garcia-Masso, Rogers, et al., 2012; Colado \& Triplett, 2008; Martins et al., 2015). However, we could not find any previous experimental intervention programs comparing the effects of traditional devices (such as free weights [FWs] or machines) with novel devices (e.g., multifunctional training with elastic tubes) during muscular strength training.
Therefore, the possible metabolic and functional adaptive effects of these novel types of strength training programs (STPs) in sedentary women aged $40-50$ years with MS remain unknown.

Recent studies have confirmed that STPs using elastic tubes can create the same levels of muscle activation in both the trunk and the extremities as machines or FWs (Aboodarda, Hamid, Muhamed, Ibrahim, \& Thompson, 2013; Jakobsen, Sundstrup, Andersen, Aagaard, \& Andersen, 2013). In addition, STPs using elastic tubes may provoke higher intrinsic motivation among health practitioners advocating strength exercises

\footnotetext{
${ }^{1}$ Austral University of Chile, Valdivia, Chile

${ }^{2}$ Research Group in Sport and Health, University of Valencia, Valencia, Spain

${ }^{3}$ Nursing Department, Faculty of Nursing and Chiropody, University of Valencia, Valencia, Spain

${ }^{4}$ Professional Institute in Therapy and Humanities (IPETH), Puebla de Zaragoza, Mexico

${ }^{5}$ Department of Physical Education and Sports, University of Valencia, Valencia, Spain
}

\section{Corresponding Author:}

Juan C. Colado, PhD, Department of Physical Education and Sports, University of Valencia, C/ Gascó Oliag 3, 46010 Valencia, Spain.

Email: juan.colado@uv.es 
Table I. Baseline Descriptive Statistics of the Anthropometric and Cardiovascular Risk Variables.

\begin{tabular}{lrr}
\hline Variable & \multicolumn{1}{c}{$M$} & \multicolumn{1}{c}{$S D$} \\
\hline Body weight $(\mathrm{kg})$ & $77.0 \mathrm{I}$ & 14.14 \\
Height $(\mathrm{cm})$ & 154.14 & 6.31 \\
Body mass index $\left(\mathrm{kg} / \mathrm{m}^{2}\right)$ & 32.58 & 5.05 \\
Percentage of fat mass & 32.31 & 5.35 \\
Waist circumference $(\mathrm{cm})$ & 95.35 & 9.21 \\
Waist/hip ratio & 0.87 & 0.05 \\
Triglycerides $(\mathrm{mg} / \mathrm{dl})$ & 150.05 & 76.32 \\
Fasting glucose $(\mathrm{mg} / \mathrm{dl})$ & 110.10 & 49.58
\end{tabular}

Note. $\mathrm{N}=62$

(Polyte, Belando, Huéscar, \& Moreno-Murcia, 2015). More specifically, José and Dal Corso (2016) recently suggested that training with elastic materials is ideal in health-care settings because they are as effective as traditional weights but much easier to transport and storage (in settings that often have very limited space) and significantly less expensive. Additionally, patients can easily acquire and use these low-cost, user-friendly materials in their own homes.

Several studies that incorporated the use of elastic tubes have demonstrated the efficacy of strength training in reducing cardiovascular risk and dynapenia in both young and menopausal women (Colado, Garcia-Masso, Rogers, et al., 2012; Colado \& Triplett, 2008; Colado, Triplett, Tella, Saucedo, \& Abellán, 2009). These studies aimed to control the participants' perception of effort by setting the range of target repetitions. Importantly, nursing units can easily incorporate training in these types of programs in both primary and secondary health care. However, relatively little is known about the effects of STPs with elastic materials at the premenopausal stage.

Against this backdrop, the objective of this study was to analyze the effects that STPs with different resistance equipment have on various metabolic and functional parameters in premenopausal women in a randomized clinical trial. Our subjects were participating in a program aimed at preventing complications associated with menopause that had been developed by a multidisciplinary group of midwives, nurses, and professionals specializing in physical education and sports. We hypothesized that implementation of an STP using a multifunctional training station that included elastic tubing (ET) would reduce cardiovascular risk and improve lipid profiles and functional fitness to the same degree as a FW STP in a cohort of sedentary premenopausal women.

\section{Materials and Method}

\section{Participants}

Participants comprised 62 women aged $40-50$ years ( $46.47 \pm$ 3.71 years; Table 1 ). All of them met the criteria to be categorized as premenopausal (Burger, Hale, Robertson, \& Dennerstein, 2007), were sedentary, did not work outside the home, had MS (Marquezine, Oliveira, Pereira, Krieger, \& Mill, 2008), and attended the Municipal Family Health Center in Valdivia (Chile). We randomly divided participants into three groups: (1) the experimental group (EG) using elastic tubes (ETG) comprising 22 participants, (2) the EG using FWs (bars and discs; FWG) with 20 participants, and (3) the control group (CG) comprising 20 participants who did not follow any STP and received no interventions. The exclusion criteria were (a) the presence of cardiovascular disease contraindicating exercise; (b) receipt of treatment to control any cardiovascular, metabolic, or musculoskeletal risk factors; (c) failure to attend the sessions scheduled in the study. As in previous studies (Colado, Garcia-Masso, Rogers, et al., 2012; Colado et al., 2009), we instructed all participants to refrain from modifying their behavior or diet and doing any other type of physical exercise for the duration of the study. To ensure strict compliance with participation instructions, we monitored these aspects of the study weekly by asking the women to complete a daily diary of activities and diet. The ethics committee at the Chilean Ministry of Health approved the research project, and the interdisciplinary council in Valdivia (Chile) granted permission. Participants signed an informed consent form and participated on a voluntary basis.

\section{Variables and Procedures}

Anthropometry. We performed anthropometric tests to describe the sample prior to administering the functional aptitude tests (Guillén del Castillo \& Linares, 2002). We used an eight-polar bioelectrical impedance system (BC-418, Tanita Corp., Tokyo, Japan) to determine fat mass percentage and body weight (Colado \& Triplett, 2008; Colado et al., 2009). We measured height and waist circumference according to International Society for the Advancement in Kinanthropometry recommendations (Stewart \& Sutton, 2012).

Metabolic parameters. A certified clinical laboratory assayed the metabolic health variables using standard techniques with 12-hr fasting blood samples. Qualified nurses withdrew the blood samples according to the procedures established by health authorities. They performed venipuncture using 32-gauge disposable hypodermic needles attached to disposable $10-\mathrm{ml}$ plastic syringes and then secured sterile gauze soaked in 96-degree ethyl alcohol to the venipuncture site with compression tape.

An automated system (Roche, Cobas C-311 analyzer) was used to analyze the chemical blood parameters. The lipid profile (total cholesterol, low-density lipoprotein cholesterol [LDL-c], and triglycerides) was analyzed using an enzymatic colorimetric test with a Shimadzu UV-160 dual beam model spectrophotometer. An immunoturbidimetry test was used to determine levels of C-reactive protein, glucose, and glycosylated hemoglobin ( $\mathrm{HbA} 1 \mathrm{c})$ biomarkers (BMs) using the automatic biochemistry and immunoturbidimetric BT 3500 analyzer. Enzyme Type 1 reagents were used in all of these 
Table 2. Exercises Used in Each of the Sessions.

\begin{tabular}{lc}
\hline Upper Limb training & Lower Limb Training \\
\hline Horizontal chest press on fitball & Front lunge \\
Biceps curl & Lateral lunge \\
Horizontal French press on fitball & Half squat \\
Military press sitting on fitball & - \\
Vertical rowing & - \\
Inclined rowing & - \\
Reverse fly & - \\
\hline
\end{tabular}

tests, and all the parameters were evaluated and analyzed before and after the intervention.

Functional fitness parameters. Although gold standard laboratory-based measurements are most desirable for assessing fitness parameters, some field tests have established reliability and validity for use in particular types of subjects and age-groups to assess physical performance variables (Cancela, Ayán, Gutiérrez-Santiago, Prieto, \& Varela, 2012; Rikli \& Jones, 1999). In the present study, we used field tests that had been previously validated.

We measured flexibility in centimeters using the sit-andreach test as described by Lemmink, Kemper, Greef, Rispens, and Stevens (2003). For balance, we recorded the number of supports and falls for the unipedal dominant-leg balance test described by Lopez (2002). To assess general coordination, we measured gait and balance in seconds using the 3-min timed up-and-go test (Bischoff et al., 2003). We used the half-squat test with a load cell to measure maximal voluntary isometric strength of the lower limbs (Dugan, La Doyle, Humphries, Hasson, \& Newton, 2004). We used a similar protocol to evaluate the maximal voluntary isometric strength of the upper limbs with the upright row test (Smith \& Loschner, 2002). We recorded strength endurance of the front and back of the trunk musculature in seconds using the prone bridge test and side bridge test (Bliss \& Teeple, 2005). For evaluation of aerobic capacity in meters, we used the 6-min walk test (Casanova et al., 2011). We evaluated and analyzed all parameters before and after the intervention.

\section{Experimental Design and Training Protocol}

We designed a standard STP and applied it equally to the ETG and FWG across the 12-week intervention (which involved 3-4 sessions per week, with 3-4 sets of 10-15 repetitions per exercise). The first 2 months of the program focused on developing local muscular endurance resistance, and the last month focused on developing muscle hypertrophy. Sessions were organized in a circuit of 10 specific exercises for upper limbs, lower limbs, and lumbo-pelvic stability (Table 2). We conducted three familiarization sessions prior to starting the program to ensure proper and safe execution with optimal adaptation to the intensity of each workout. We also designed a warm-up (lasting a maximum of $10 \mathrm{~min}$ ) that included a light trot, joint mobility exercises, and stretching. We controlled the intensity for the elastic-tubing exercises from the beginning to the end of the program using a Resistance Perceived Effort Scale (Colado, Garcia-Masso, Triplett, et al., 2012) with an incremental range of 7-9. We allowed $30 \mathrm{~s}$ of active between exercises (jogging in place and soft tissue joint mobility for upper limbs) and $60 \mathrm{~s}$ recovery between sets (completion of the whole circuit). Nurses and technicians with extensive experience with physical activity supervised the exercises at all times.

\section{Exercise Equipment}

The ETG used a multifunction training station $(111.8 \mathrm{~cm}$ long, $61 \mathrm{~cm}$ wide, and $5.1 \mathrm{~cm}$ high) with different types of elastic tubes anchored to it in several positions (TheraBand Exercise Station, Hygienic Corporation, Akron, $\mathrm{OH}$ ). This training station also had anchor points for affixing elastic tubes with up to three different levels of viscoelastic hardness $(30.5 \mathrm{~cm}$ of length) that could be attached to either individual handles or a bar. We developed the prescribed exercises by modifying the types of anchor and material and by adding a fitball. The FWG used bars, discs, and standard dumbbells, and as with the ETG STP, we used a fitball for assistance in certain exercises. The intensity was modified while the participant did the exercise by changing the equipment according to the procedure followed in previous studies (Colado et al., 2009, 2010; Colado \& Triplett, 2008).

\section{Statistical Analysis}

We analyzed the data using the Statistical Program for the Social Sciences (SPSS, version 20.0; SPSS Inc., Chicago, IL). All the variables met the assumption of normality (Kolmogorov-Smirnov test) and homoscedasticity (Levene test). We used standard statistical methods to calculate the mean and standard deviation as a measurement of the central tendency and dispersion, respectively. To establish the effects of the training program on the dependent variables, we applied an analysis of variance of repeated one-way measurements. When we found differences, we applied post hoc Bonferroni analysis. A $95 \%$ confidence level (significance of $p<.05$ ) was accepted for all the analyses.

\section{Results}

There were no significant differences in the blood BMs or functional parameters before the start of the intervention.

\section{Effects of the Intervention on Blood BMs}

C-reactive protein level decreased significantly from baseline to after the intervention in both the ETG and FWG $(p<.05)$ and also compared with the control group ( $p<.05$; Table 3$)$. HbA1c was significantly reduced in both EGs and significantly augmented in the $\mathrm{CG}$, although we did not find significant between-group differences. We found a similar trend for the CG for the other metabolic parameters (not statistically 
Table 3. Pre and Postintervention Metabolic Parameter Results by Study Group.

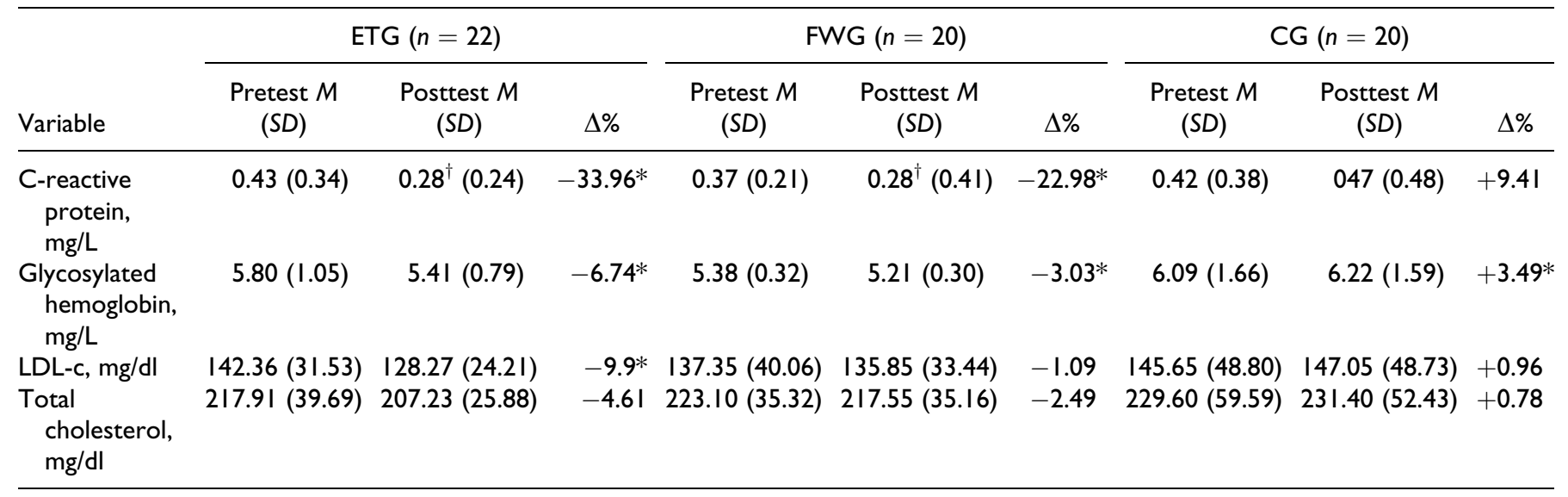

Note. $\Delta \%=$ percentage difference between pre- and posttest; CG = control group; ETG = elastic-tube training group; FWG $=$ free-weight training group; LDL-c $=$ low-density lipoprotein cholesterol; $M=$ median; $S D=$ standard deviation.

${ }^{*} p<.05$, statistically significant intragroup difference from pre- to posttest. ${ }^{\dagger} p<.05$, statistically significant difference relative to the control group.

significant) at the end of the intervention period. For the ETG, we also found a significant reduction in LDL-c $(p<.05)$ from baseline to postintervention as well as a trend toward reduction in all of the metabolic parameters (not statistically significant). There were no statistically significant within- or betweengroup differences in total cholesterol after the training program intervention. There were no significant differences between the two EGs in blood BMs.

\section{Effects of the Intervention on the Functional Parameters}

All of the functional variables analyzed (Table 4) showed improved intragroup values in both the ETG and FWG $(p<$ $.05)$. An increase in physical performance ability with respect to the CG was also noted in each of the EGs, and there was no significant difference between the ETG and FWG. In other words, there was a significant increase in general coordination, maximal voluntary isometric strength of the upper and lower limbs, the local muscular endurance of the lateral and ventral trunk area, and aerobic capacity $(p<.05)$ in the EGs relative to the control group. None of these functional skills significantly changed in the $\mathrm{CG}$.

\section{Discussion}

In the present study, we evaluated the effects of a physical exercise STP with an innovative device, specifically the elastic-tube multifunction training station, compared to those of an STP with traditional FW devices. We found improvements in blood BMs and functional aptitude parameters in premenopausal women with MS in both exercise groups after the intervention, thus confirming our initial hypothesis. Our results provide evidence that training with elastic materials produces improvements in both metabolic parameters and functional fitness (Paditsaeree, Intiraporn, \& Lawsirirat, 2014) equal to those achieved using other traditional devices such as FWs (Colado et al., 2009, 2010).
Previous studies have shown that moderate- to highintensity exercise produces improvements in insulin sensitivity, fat oxidation, and triglyceride and LDL-c concentrations in sedentary overweight or obese adults (Gondim et al., 2015; Whyte, Ferguson, Wilson, Scott, \& Gill, 2013). Of note, there was a significant decrease in $\mathrm{HbA1c}$ in both EGs in the present study. While LDL-c level decreased significantly by $9.9 \%$ in the ETG and nonsignificantly by $1.09 \%$ in the FWG from preto postintervention, it increased nonsignificantly by $0.96 \%$ in the CG. This finding contrasts with that of Behall, Howe, Martel, Scott, and Dooly (2003) who did not find any differences in LDL-c levels after groups of overweight and sedentary pre- or postmenopausal women performed exercise for 3 months (aerobic or strength). Similarly, Rodríguez et al. (2005) found no changes in lipid profiles after an STP in sedentary adults with high cholesterol. However, our results agree with other work that indicated that STP improved the lipid profile of premenopausal women, reducing both total cholesterol and LDL-c and increasing high-density lipoprotein cholesterol (HDL-c; Prabhakaran, Dowling, Branch, Swain, \& Leutholtz, 1999). In a study analogous to our own, Park et al. (2015) recently examined how STP with elastic bands affected glucose, body composition, and physical fitness (isometric strength of upper and lower limbs measured as biceps and squat curls) in adult women with type 2 diabetes. After 12 weeks of intervention, all variables were significantly improved. Likewise, postmenopausal women who participated in an exercise program similar to the program in the present study achieved significant improvements in total cholesterol/HDL-c ratio and a reduction in triglyceride and LDL-c levels (Colado et al., 2009).

In addition, both groups participating in the STP in the present study had significantly reduced C-reactive protein levels by an average of $28.47 \%$ compared to the CG. This finding is noteworthy because $\mathrm{C}$-reactive protein is one of the $\mathrm{BMs}$ that help to define cardiovascular risk and systemic inflammation (Fischer, Berntsen, Perstrup, Eskildsen, \& Pedersen, 2007). Previous researchers have reported comparable findings for 


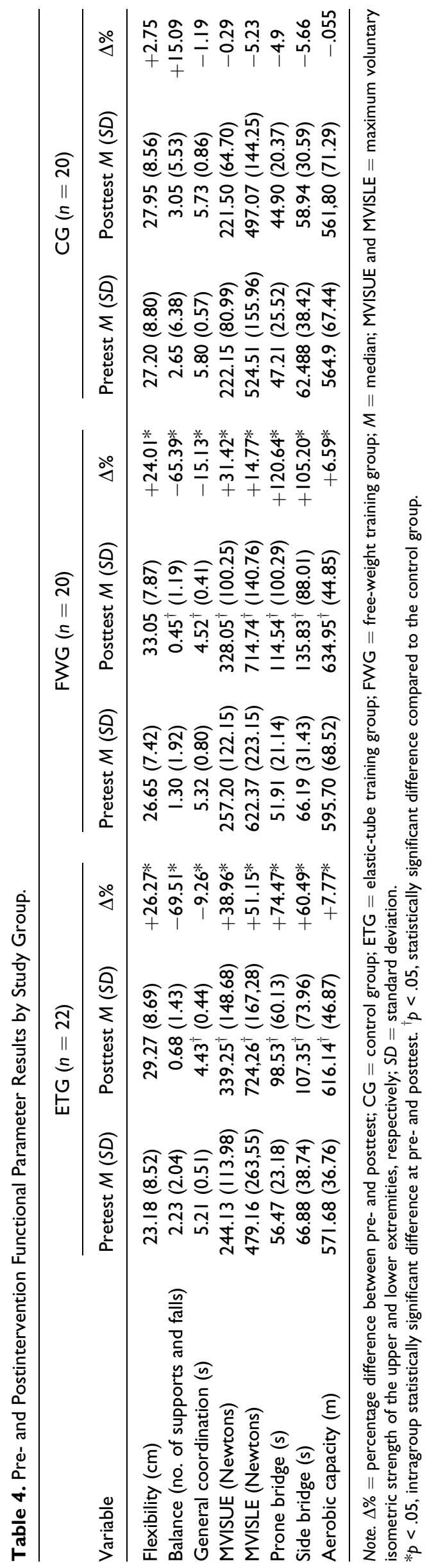


similar programs in different age-groups (Donges, Duffield, \& Drinkwater, 2010; Phillips et al., 2012). However, very few scientific studies have specifically measured the changes that occur in C-reactive protein level after implementing an STP specifically with elastic materials, and the few that have mostly found no positive effects (K. Kim, Lee, Jeon, Jeong, \& Kye Soon, 2010; So et al., 2013). This absence of response may be because there were no precise controls on the intensity of the elastic tube or band training (Martins et al., 2015). Nevertheless, the present study provides evidence that the use of appropriate training methodology, with either elastic tubes or traditional materials such as FWs, can reduce cardiovascular risk and systemic inflammation.

Both types of training devices were also effective in producing positive functional adaptations; however, there were some differences between them. The anchored elastic tube device resulted in $36.4 \%$ greater strength adaptations of the lower extremities, while FW resulted in greater local muscular endurance of the trunk. These differences were not significant; however, they are in line with recent findings about stability and body positioning when handling the resistance during strength exercises (Behm \& Colado, 2012). These findings suggest that variation in training devices during exercise programs might help to maximize physical adaptations (Colado et al., 2011).

This study did have some limitations that future researchers should consider and address in subsequent studies. For one thing, a longer intervention might have allowed for the detection of significant differences between the EGs in both the functional and metabolic values. It would also be interesting to compare anthropometric parameters at the start and end of the program. Similarly, causative factors of MS, continence, and bone mineral density could also be analyzed. Longitudinal studies are needed to analyze additional biomedical parameters and physical and psychosocial variables related to the performance of STPs using alternative materials; these should focus on improving the quality of life and well-being of premenopausal women.

\section{Conclusion}

In the present study, we demonstrated that a 12-week STP using a multifunctional training station with elastic tubes improved the metabolic health and functional aptitude of premenopausal women with moderate cardiovascular risk (i.e., with MS) to a similar degree as did an STP using traditional FW equipment. Importantly, these nontraditional devises based on ET are highly practical in that they are low cost, accessible, effective, and motivating. Qualified health personnel could readily use these materials to develop programs to improve patient strength and health risk factors and easily supervise such programs (Colado \& Triplett, 2008). Our findings thus provide scientific evidence, supporting the use of practical tools that health professionals could use to help prevent and treat cardiovascular and metabolic diseases in sedentary premenopausal women. In addition, the study demonstrates collaborative strategies that can be used between different health and nonhealth professionals to help prevent disease and improve patients' quality of life. Nurses, in particular, can play a key role in coordinating physical exercise interventions.

\section{Authors' Contribution}

Jorge Flandez contributed to conception, design, and acquisition; drafted the manuscript; critically revised the manuscript for important intellectual content; gave final approval; and agrees to be accountable for all aspects of work ensuring integrity and accuracy. Noelia Belando contributed to conception and design; drafted the manuscript; gave final approval; and agrees to be accountable for all aspects of work ensuring integrity and accuracy. Pedro Gargallo contributed to conception and design; drafted the manuscript; gave final approval; and agrees to be accountable for all aspects of work ensuring integrity and accuracy. Julio Fernández-Garrido contributed to conception and design; critically revised the manuscript for important intellectual content; gave final approval; and agrees to be accountable for all aspects of work ensuring integrity and accuracy. Ronald A. VargasFoitzick contributed to conception and design; critically revised the manuscript for important intellectual content; gave final approval; and agrees to be accountable for all aspects of work ensuring integrity and accuracy. Jose Devis-Devis contributed to conception and design; critically revised the manuscript for important intellectual content; gave final approval; and agrees to be accountable for all aspects of work ensuring integrity and accuracy. Juan C. Colado contributed to conception, design, analysis, and interpretation of data; drafted the manuscript; critically revised the manuscript for important intellectual content; gave final approval; and agrees to be accountable for all aspects of work ensuring integrity and accuracy.

\section{Declaration of Conflicting Interests}

The author(s) declared no potential conflicts of interest with respect to the research, authorship, and/or publication of this article.

\section{Funding}

The author(s) received no financial support for the research, authorship, and/or publication of this article.

\section{References}

Aboodarda, S. J., Hamid, M. S. A., Muhamed, A. M. C., Ibrahim, F., \& Thompson, M. (2013). Resultant muscle torque and electromyography activity during high intensity elastic resistance and free weight exercises. European Journal of Sport Science, 13, 155-163. doi:10.1080/17461391.2011.586438

Behall, K. M., Howe, J. C., Martel, G., Scott, W. H., \& Dooly, C. R. (2003). Comparison of resistive to aerobic exercise training on cardiovascular risk factors of sedentary, overweight premenopausal and postmenopausal women. Nutrition Research, 23, 607-619. doi:10.1016/S0271-5317(03)00015-0

Behm, D., \& Colado, J. C. (2012). The effectiveness of resistance training using unstable surfaces and devices for rehabilitation. International Journal of Sports Physical Therapy, 7, 226-241.

Bischoff, H. A., Stähelin, H. B., Monsch, A. U., Iversen, M. D., Weyh, A., Von, D., ... Theiler, R. (2003). Identifying a cut-off point for normal mobility: A comparison of the timed 'up and go' test in community-dwelling and institutionalised elderly women. Age and Ageing, 32, 315-320. doi:10.1093/ageing/32.3.315 
Bliss, L. S., \& Teeple, P. (2005). Core stability: The centerpiece of any training program. Current Sports Medicine Reports, 4, 179-183. doi:10.1093/ageing/32.3.315

Burger, H. G., Hale, G. E., Robertson, D. M., \& Dennerstein, L. (2007). A review of hormonal changes during the menopausal transition: Focus on findings from the Melbourne Women's Midlife Health Project. Human Reproduction Update, 13, 559-565. doi:10.1093/humupd/dmm020

Cancela, J. M., Ayán, C., Gutiérrez-Santiago, A., Prieto, I., \& Varela, S. (2012). The senior fitness test as a functional measure in Parkinson's disease: A pilot study. Parkinsonism and Related Disorders, 18, 170-173. doi:10.1016/j.parkreldis.2011.09.016

Casanova, C., Celli, B. R., Barria, P., Casas, A., Cote, C., De Torres, J., ... Aguirre-Jaime, A. (2011). The 6-min walk distance in healthy subjects: Reference standards from seven countries. European Respiratory Journal, 37, 150-156. doi:10. 1183/09031936.00194909

Clark, B. C., \& Manini, T. M. (2008). Sarcopenia y dynapenia. Journals of Gerontology Series A: Biological Sciences and Medical Sciences, 63, 829-834. doi:10.1093/gerona/63.8.829

Colado, J. C., Garcia-Masso, X., Pellicer, M., Alakhdar, Y., Benavent, J., \& Cabeza-Ruiz, R. (2010). A comparison of elastic tubing and isotonic resistance exercises. International Journal of Sports Medicine, 31, 810-817. doi:10.1055/s-0030-1262808

Colado, J. C., Garcia-Masso, X., Rogers, M. E., Tella, V., Benavent, J., $\&$ Dantas, E. H. (2012). Effects of aquatic and dry land resistance training devices on body composition and physical capacity in postmenopausal women. Journal of Human Kinetics, 32, 185-195. doi:10.2478/v10078-012-0035-3

Colado, J. C., Garcia-Masso, X., Triplett, T. N., Flandez, J., Borreani, S., \& Tella, V. (2012). Concurrent validation of the OMNIResistance Exercise Scale of Perceived Exertion with TheraBand resistance bands. Journal of Strength and Conditioning Research, 26, 3018-3024. doi:10.1519/JSC.0b013e318245c0c9

Colado, J. C., Pablos, C., Chulvi-Medrano, I., Garcia-Masso, X., Flandez, J., \& Behm, D. G. (2011). The progression of paraspinal muscle recruitment intensity in localized and global strength training exercises is not based on instability alone. Archives of Physical Medicine and Rehabilitation, 92, 1875-1883. doi:10.1016/j.apmr. 2011.05.015

Colado, J. C., \& Triplett, N. T. (2008). Effects of a short-term resistance program using elastic bands versus weight machines for sedentary middle-aged women. Journal of Strength and Conditioning Research, 22, 1441-1448. doi:10.1519/JSC. 0b013e31817ae67a

Colado, J. C., Triplett, N. T., Tella, V., Saucedo, P., \& Abellán, J. (2009). Effects of aquatic resistance training on health and fitness in postmenopausal women. European Journal of Applied Physiology, 106, 113-122. doi:10.1007/s00421-009-0996-7

Donges, C. E., Duffield, R., \& Drinkwater, E. J. (2010). Effects of resistance or aerobic exercise training on interleukin-6, C-reactive protein, and body composition. Medicine and Science in Sports and Exercise, 42, 304-313. doi:10.1249/MSS.0b013e3181b117ca

Dugan, E. L., La Doyle, T., Humphries, B., Hasson, C. J., \& Newton, R. U. (2004). Determining the optimal load for jump squats: A review of methods and calculations. Journal of Strength and
Conditioning Research, 18, 668-674. doi:10.1519/00124278200408000-00050

Fischer, C. P., Berntsen, A., Perstrup, L. B., Eskildsen, P., \& Pedersen, B. K. (2007). Plasma levels of interleukin-6 and C-reactive protein are associated with physical inactivity independent of obesity. Scandinavian Journal of Medicine \& Science in Sports, 17, 580-587. doi:10.1111/j.1600-0838.2006.00602.x

Gondim, O. S., De Camargo, V. T. N., Gutierrez, F. A., De Oliveira, P. F., Passos, M. E. P., Momesso, C. M., ... Cury-Boaventura, M. F. (2015). Benefits of regular exercise on inflammatory and cardiovascular risk markers in normal weight, overweight and obese adults. PLoS One, 10, e0140596. doi:10.1371/journal.pone.0140596

Gudmundsdottir, S. L., Flanders, W. D., \& Augestad, L. B. (2013). Physical activity and cardiovascular risk factors at menopause: The Nord-Trøndelag health study. Climacteric, 16(4), 438-446. doi:10. 3109/13697137.2013.768231

Guillén del Castillo, M., \& Linares, D. (2002). Bases biológicas y fisiológicas del movimiento humano. Madrid, Spain: Editorial Médica panamericana.

Huebschmann, A. G., Kohrt, W. M., Herlache, L., Wolfe, P., Daugherty, S., Reusch, J. E., ... Regensteiner, J. G. (2015). Type 2 diabetes exaggerates exercise effort and impairs exercise performance in older women. Open Diabetes Research and Care, 3, e000124. doi:10.1136/ bmjdrc-2015-000124

Jakobsen, M. D., Sundstrup, E., Andersen, C. H., Aagaard, P., \& Andersen, L. L. (2013). Muscle activity during leg strengthening exercise using free weights and elastic resistance: Effects of ballistic vs controlled contractions. Human Movement Science Journal, 32, 65-78. doi:10.1016/j.humov.2012. 07.002

José, A., \& Dal Corso, S. (2016). Inpatient rehabilitation improves functional capacity, peripheral muscle strength and quality of life in patients with community-acquired pneumonia: A randomised trial. Journal of Physiotherapy, 62, 96-102. doi:10.1016/ j.jphys. 2016.02.014

Kim, J. W., \& Kim, D. Y. (2012). Effects of aerobic exercise training on serum sex hormone binding globulin, body fat index and metabolic syndrome factors in obese postmenopausal women. Metabolic Syndrome and Related Disorders, 10, 452-457. doi:10. 1089/met.2012.0036

Kim, K., Lee, H., Jeon, C., Jeong, J., \& Kye Soon, P. (2010). The effect of elastic band exercise on atherosclerosis indices and $\mathrm{C}$ reactive protein of disabled people with spinal cord injury. Journal of Exercise Rehabilitation, 6, 99-107.

Lauretani, F., Russo, C. R., Bandinelli, S., Bartali, B., Cavazzini, C., \& Di Iorio, A., ... Ferrucci, L. (2003). Age-associated changes in skeletal muscles and their effect on mobility: An operational diagnosis of sarcopenia. Journal of Applied Physiology, 95, 1851-1860. doi:10.1152/japplphysiol.00246.2003

Lemmink, K. A., Kemper, H. C., Greef, M. H., Rispens, P., \& Stevens, M. (2003). The validity of the sit-and-reach test and the modified sit-and-reach test in middle-aged to older men and women. Research Quarterly for Exercise and Sport, 74, 331-336. doi:10. 1080/02701367.2003.10609099

López, E. J. M. (2002). Physical fitness tests. Barcelona, Spain: Paidotribo Editorial. 
Marquezine, G. F., Oliveira, C. M., Pereira, A. C., Krieger, J. E., \& Mill, J. G. (2008). Metabolic syndrome determinants in an urban population from Brazil: Social class and genderspecific interaction. International Journal of Cardiology, 129, 259-265.

Martins, W. R., Safons, M. P., Bottaro, M., Blasczyk, J. C., Diniz, L. R., Fonseca, R. M., ... de Oliveira, R. J. (2015). Effects of short term elastic resistance training on muscle mass and strength in untrained older adults: A randomized clinical trial. BMC Geriatrics, 15, 99. doi:10.1186/s12877015-0101-5

Orsatti, F. L., Nahas, E. A., Maesta, N., Nahas-Neto, J., \& Burini, R. C. (2008). Plasma hormones, muscle mass and strength in resistance-trained postmenopausal women. Maturitas, 59, 394-404. doi:10.1016/j.maturitas.2008.04.002

Paditsaeree, K., Intiraporn, C., \& Lawsirirat, C. (2014). Comparison between the effects of combining elastic and free-weight resistance and free-weight resistance on force and power production. Journal of Strength and Conditioning Research. Advance online publication. doi:10.1519/JSC.000000 0000000459

Park, B. S., Khamoui, A., Brown, L. E., Kim, D. Y., Han, K., Min, K. W., \& An, G. (2015). Effects of elastic band resistance training on glucose control, body composition, and physical function in women with short- vs. long-duration Type 2 diabetes. Journal of Strength and Conditioning Research. doi:10.1519/JSC. 00000000001256

Phillips, M. D., Patrizi, R. M., Cheek, D. J., Wooten, J. S., Barbee, J. J., \& Mitchell, J. B. (2012). Resistance training reduces subclinical inflammation in obese, postmenopausal women. Medicine and Science in Sports and Exercise, 44, 2099-2110. doi:10.1249/MSS. 0b013e3182644984
Polyte, P. J. J., Belando, N., Huéscar, E., \& Moreno-Murcia, J. A. (2015). Effect of style teaching in the motivation of women practitioners of physical exercise. Acción Psicológica, 12, 57-64. doi:10. 5944/ap.12.1.13977

Prabhakaran, B., Dowling, E. A., Branch, J. D., Swain, D. P., \& Leutholtz, B. C. (1999). Effect of 14 weeks of resistance training on lipid profile and body fat percentage in premenopausal women. British Journal of Sports Medicine, 33, 190-195. doi:10.1136/ bjsm.33.3.190

Rikli, R. E., \& Jones, J. (1999). Development and validation of a functional fitness test for community-residing older adults. Journal of Aging and Physical Activity, 7, 129-161.

Rodríguez, J., Vieira, M., Pacheco, M. E., De Mata Silva, L. G., Grubert, C. S., \& Simoes, H. G. (2005). Treinamento resistido de oito semanas melhora a aptidão física mas não altera o perfil lipídico de indivíduos hipercolesterolêmicos. Lecturas: Educación Fisica y Deportes, 81. Retrieved from http://www.efdeportes. com/efd81/lipidos.htm

Smith, R. M., \& Loschner, C. (2002). Biomechanics feedback for rowing. Journal of Sports Sciences, 20, 783-791. doi:10.1080/ 026404102320675639

So, W. Y., Song, M., Park, Y. H., Cho, B. L., Lim, J. Y., Kim, S. H., \& Song, W. (2013). Body composition, fitness level, anabolic hormones, and inflammatory cytokines in the elderly: A randomized controlled trial. Aging Clinical and Experimental Research, 25, 167-174. doi:10.1007/s40520-013-0032-y

Stewart, A., \& Sutton, L. (2012). Body composition in sport, exercise, and health. New York, NY: Routledge.

Whyte, L. J., Ferguson, C., Wilson, J., Scott, R. A., \& Gill, J. M. (2013). Effects of single bout of very high-intensity exercise on metabolic health biomarkers in overweight/obese sedentary men. Metabolism, 62, 212-219. doi:10.1016/j.metabol.2012.07.019 